\title{
INVERSE SPECTRAL-SCATTERING PROBLEM FOR THE STURM-LIOUVILLE OPERATOR ON A NONCOMPACT STAR-TYPE GRAPH
}

\author{
S. A. BUTERIN AND G. FREILING
}

\begin{abstract}
We study the Sturm-Liouville operator on a noncompact star-type graph consisting of a finite number of compact and noncompact edges under standard matching conditions in the internal vertex. We introduce and investigate the so-called spectralscattering data, which generalize the classical spectral data for the Sturm-Liouville operator on the half-line and the scattering data on the line. Developing the idea of the method of spectral mappings we prove that the specification of the spectral-scattering data uniquely determines the Sturm-Liouville operator on the graph.
\end{abstract}

\section{Introduction}

We study an inverse problem for the Sturm-Liouville differential operator on an arbitrary noncompact star-type graph possessing a finite number of edges. This inverse problem consists in recovering the potential of the Sturm-Liouville operator on the graph along with the coefficients of boundary conditions in boundary vertices from appropriate spectral characteristics. Differential operators on graphs (spatial networks) often appear in mathematics, mechanics, physics, geophysics, physical chemistry, biology, electronics, nanoscale technology and other branches of natural sciences and engineering (see [1]-[14]). Recently there has increased interest in spectral theory of Sturm-Liouville or Schrödinger operators on graphs (for a good review of such publications see [15], [16]). Most of the works in this direction are devoted to the so-called direct problems of studying properties of the spectrum and the root functions. Inverse spectral problems on graphs are because of their nonlinearity more difficult and their intensive investigation started only several years ago.

Most complete results on inverse spectral problems for the Sturm-Liouville operator on graphs were obtained for compact graphs including trees and also graphs with cycles (see

Corresponding author: S. A. Buterin.

2010 Mathematics Subject Classification. 34A55, 34B45, 34B40, 47E05.

Key words and phrases. Differential equations on graphs, noncompact graphs, inverse scattering problems, inverse spectral problems, method of spectral mappings.

This research was supported in part by Grant 13-01-00134 of RFBR and by joint program "Mikhail Lomonosov" of Ministry of Education and Science of Russian Federation and DAAD (project 15007). 
[17]-[23] and the references therein). Certain systems of spectra or Weyl functions were shown to be appropriate input data in the compact case. In [24] an inverse spectral problem was studied for the Sturm-Liouville operator on a noncompact tree with a single noncompact edge, where Weyl functions associated with finite boundary vertices were introduced and used as the spectral data. Inverse spectral problems for higher-order differential operators on compact trees were studied in [25] and on trees with a single noncompact edge in [26].

In the case of graphs with more than one noncompact edges it is natural to use the socalled scattering data, which generalize the scattering data for the Sturm-Liouville operators on the line [27]-[31]. For the first time the inverse scattering problem on a noncompact graph was discussed in [32], but only for a very special case of star-type graphs without compact edges. In [33] this case was reduced to the inverse scattering problem for the matrix SturmLiouville operator on the half-line with a special selfadjoint boundary condition in the origin. In [34]-[36] inverse scattering problems were studied for graphs consisting of a cycle and a finite number rays.

In presence of compact boundary edges the scattering data are not sufficient to determine the potential on all edges of the graph. For example, in [37], [38] some non-uniqueness results were obtained for inverse scattering problems on general noncompact graphs.

In this paper we provide a formulation and prove the uniqueness theorem for the inverse problem of recovering the Sturm-Liouville operator on a noncompact star-type graph possessing compact edges. In this situation it is natural to consider a mixture of inverse spectral and inverse scattering problems. The spectral-scattering data, which we use, can be subdivided into two parts. The "spectral" part consists of the so-called Weyl functions associated with the compact edges, which generalize the Weyl function of the Sturm-Liouville operator on the half-line (see, e.g., [31]) and on a noncompact tree with a single infinite edge (see [24]). The "scattering" part, in turn, includes a portion of negative eigenvalues together with the so-called reflection coefficients and norming constants associated with all but one noncompact edges. Such scattering data generalizes the left (or right) scattering data for the SturmLiouville operator on the line (see [27]-[31]). Since some eigenvalues are poles of the Weyl functions from the "spectral" part, in the "scattering" part it suffices to specify only the socalled invisible from compact edges eigenvalues. Developing the ideas of the method of spectral mappings [39], we prove that the specification of these spectral-scattering data uniquely determines the potential of the Sturm-Liouville operator on the graph along with the coefficients of the boundary conditions in the boundary vertices. We note that the case without compact edges was studied separately in [40] and also with general matching conditions in [41]. The presence of compact edges causes, inter alia, new qualitative difficulties in the investigation of inverse problems due to a more complicated behavior of the scattering solutions and reflection coefficients. 
In the next section we introduce and investigate special solutions of the differential equation on the graph, which possess necessary asymptotical and analytical properties. In Section 3 properties of spectral characteristics are studied and the spectral-scattering data are introduced. In Section 4 we prove the uniqueness theorem of the inverse problem.

\section{Special solutions}

In a finite-dimensional Euclidean space we consider a noncompact star-type graph $\Gamma$ with the vertices $v_{0}, v_{1}, \ldots, v_{p}$ and the edges $\varepsilon_{1}, \ldots, \varepsilon_{m}$, where $\varepsilon_{j}=\left[v_{0}, v_{j}\right], j=\overline{1, p}$, are segments and $\varepsilon_{j}, j=\overline{p+1, m}$, are rays with the common vertex $v_{0}$. Let for definiteness $p \geq 1$ and $m-p \geq 2$. Moreover, for simplicity we assume that the length of each compact edge is equal to 1 (it follows from the proofs that our method works also for arbitrary lengthes). The vertices $v_{j}, j=\overline{1, p}$, are called boundary vertices. The vertex $v_{0}$ is called internal vertex and considered as the initial point of all edges, which, in turn, are parameterized by $x \geq 0$, and $x=0$ corresponds always to the internal vertex. For the compact edges we have $x \in[0,1]$ and $x=1$ corresponds to the boundary vertex. Any function $y$ on $\Gamma$ can be represented as a vector $y=\left[y_{j}(x)\right]_{j=\overline{1, m}}$ (but not a vector-function), where the function $y_{j}(x)$ is defined on the edge $\varepsilon_{j}$. Let $q=\left[q_{j}(x)\right]_{j=\overline{1, m}}$ be a real-valued function on $\Gamma$, which we will call the potential. We assume that

$$
q_{j}(x) \in L(0,1), \quad j=\overline{1, p} ; \quad q_{j}(x), x q_{j}(x) \in L(0, \infty), j=\overline{p+1, m} .
$$

Consider the Sturm-Liouville equation on $\Gamma$ :

$$
\ell_{j} y_{j}:=-y_{j}^{\prime \prime}+q_{j}(x) y_{j}=\lambda y_{j}
$$

where $x \in(0,1)$ for $j=\overline{1, p} ; x \in(0, \infty)$ for $j=\overline{p+1, m}$ and $\lambda$ is the spectral parameter. Let the function $y=\left[y_{j}(x)\right]_{j=\overline{1, m}}$ satisfy the following $m$ matching conditions in the vertex $v_{0}$ :

$$
\left.\begin{array}{c}
y_{1}(0)=y_{j}(0), \quad j=\overline{2, m}, \quad \text { (continuity condition) } \\
\sum_{j=1}^{m} y_{j}^{\prime}(0)=0 \quad \text { (Kirchhoff's condition). }
\end{array}\right\}
$$

and the following boundary conditions in the boundary vertices:

$$
U_{j}\left(y_{j}\right):=y_{j}^{\prime}(1)+H_{j} y_{j}(1)=0, \quad j=\overline{1, p},
$$

where $H_{j} \in \mathbb{R}, j=\overline{1, p}$. Denote by $L=L(q, H)$ the boundary value problem (1)-(3), where $H=\left[H_{j}\right]_{j=\overline{1, p}}$. We also consider the corresponding operator

$$
\mathscr{L}: D(\mathscr{L}) \rightarrow L_{2}(\Gamma), \quad y=\left[y_{j}\right]_{j=\overline{1, m}} \mapsto \mathscr{L} y:=\left[\ell_{j} y_{j}\right]_{j=\overline{1, m}}
$$


where the domain of definition $D(\mathscr{L})$ consists of functions $y=\left[y_{j}(x)\right]_{j=\overline{1, m}}$ such that

$$
y_{j} \in W_{1}^{2}[0,1], \quad j=\overline{1, p} ; \quad y_{j} \in W_{1, l o c}^{2}[0, \infty), y_{j}, \ell_{j} y_{j} \in L_{2}(0, \infty), j=\overline{p+1, m},
$$

and $y$ satisfies (2), (3). The matching conditions (2) are called the standard matching conditions. In electrical circuits (2) express Kirchhoff's law, in elastic string network they express the balance of tension, etc.

Let $\lambda=\rho^{2}$. Denote

$$
\begin{aligned}
\mathbb{R}^{*} & =\mathbb{R} \backslash\{0\}, \quad \Omega_{ \pm}=\{\rho: \pm \operatorname{Im} \rho>0\}, \\
Q_{0 j}(x) & =\int_{x}^{\infty}\left|q_{j}(t)\right| d t, \quad Q_{1 j}(x)=\int_{x}^{\infty} Q_{0 j}(t) d t=\int_{x}^{\infty}(t-x)\left|q_{j}(t)\right| d t .
\end{aligned}
$$

Let $C_{j}(x, \lambda), S_{j}(x, \lambda)$ be the solutions of equation (1) on the edge $\varepsilon_{j}$ satisfying the initial conditions

$$
C_{j}(0, \lambda)=S_{j}^{\prime}(0, \lambda)=1, \quad C_{j}^{\prime}(0, \lambda)=S_{j}(0, \lambda)=0 .
$$

On the compact edges $\varepsilon_{j}, j=\overline{1, p}$, we introduce also the solutions $\psi_{j}(x, \lambda), \theta_{j}(x, \lambda)$ such that

$$
\psi_{j}(1, \lambda)=-\theta_{j}^{\prime}(1, \lambda)=1, \quad U_{j}\left(\psi_{j}(x, \lambda)\right)=\theta_{j}(1, \lambda)=0
$$

For $|\rho| \rightarrow \infty$ the following estimates hold (see, e.g., [31]):

$$
\left.\begin{array}{c}
\psi_{j}(x, \lambda)=\cos \rho(1-x)-\omega_{j}(x) \frac{\sin \rho(1-x)}{\rho}+o\left(\frac{1}{\rho} \exp (|\operatorname{Im} \rho|(1-x))\right), \\
\psi_{j}^{\prime}(x, \lambda)=\rho \sin \rho(1-x)+\omega_{j}(x) \cos \rho(1-x)+o(\exp (|\operatorname{Im} \rho|(1-x))),
\end{array}\right\}
$$

where

$$
\omega_{j}(x)=-H_{j}-\frac{1}{2} \int_{x}^{1} q_{j}(t) d t, \quad j=\overline{1, p} .
$$

The following theorem introduces the Jost solution $e_{j}(x, \rho)$ on the noncompact edges $\varepsilon_{j}, j=$ $\overline{p+1, m}$, with prescribed behaviour in $\infty$ (see [31]).

Theorem 1. Let $\rho \in \overline{\Omega_{+}}$and $j \in\{p+1, \ldots, m\}$. Equation (1) has a unique solution $y_{j}=e_{j}(x, \rho)$ satisfying the integral equation

$$
e_{j}(x, \rho)=\exp (i \rho x)+\int_{x}^{\infty} \frac{\sin \rho(t-x)}{\rho} q_{j}(t) e_{j}(t, \rho) d t .
$$

The function $e_{j}(x, \rho)$ has the following properties:

(i) For each fixed $x$ the functions $e_{j}^{(v)}(x, \rho), v=0,1$, are analytic in $\Omega_{+}$and continuous in $\overline{\Omega_{+}}$. 
(ii) $\operatorname{For} v=0,1$

$$
e_{j}^{(v)}(x, \rho)=(i \rho)^{v} \exp (i \rho x)(1+o(1)), \quad x \rightarrow+\infty,
$$

uniformly in $\overline{\Omega_{+}}$. Moreover, for $\rho \in \overline{\Omega_{+}}$

$$
\left.\begin{array}{c}
\left|e_{j}(x, \rho) \exp (-i \rho x)\right| \leq \exp \left(Q_{1 j}(x)\right), \\
\left|e_{j}(x, \rho) \exp (-i \rho x)-1\right| \leq Q_{1 j}(x) \exp \left(Q_{1 j}(x)\right), \\
\left|e_{j}^{\prime}(x, \rho) \exp (-i \rho x)-i \rho\right| \leq Q_{0 j}(x) \exp \left(Q_{1 j}(x)\right) .
\end{array}\right\}
$$

(iii) For each fixed $\rho \in \Omega_{+} e_{j}(x, \rho) \in L_{2}(0, \infty)$. Moreover, $e_{j}(x, \rho)$ is a unique solution of (1) (up to a multiplicative constant) having this property.

(iv) $\operatorname{For}|\rho| \rightarrow \infty, \rho \in \overline{\Omega_{+}}, v=0,1$

$$
e_{j}^{(v)}(x, \rho)=(i \rho)^{v} \exp (i \rho x)\left(1+\frac{\omega_{j}(x)}{i \rho}+o\left(\frac{1}{\rho}\right)\right), \quad \omega_{j}(x)=-\frac{1}{2} \int_{x}^{\infty} q_{j}(t) d t
$$

uniformly for $x \geq 0$.

(v) For $\rho \in \mathbb{R}^{*}$ the functions $e_{j}(x, \rho), e_{j}(x,-\rho)$ form a fundamental system of solutions for (1), and

$$
\left\langle e_{j}(x, \rho), e_{j}(x,-\rho)\right\rangle \equiv-2 i \rho
$$

where $\langle y, z\rangle:=y z^{\prime}-y^{\prime} z$.

The next lemma (see [31]) gives properties of the Jost solutions $e_{j, r}(x, \rho)$ related to the potentials $q_{j, r}(x)$ that approximate $q_{j}(x)$.

Lemma 1. If

$$
\lim _{r \rightarrow \infty} \int_{0}^{\infty}(1+x)\left|q_{j, r}(x)-q_{j}(x)\right| d x=0
$$

then

$$
\lim _{r \rightarrow \infty} \sup _{\rho \in \Omega_{+}} \sup _{x \geq 0}\left|\left(e_{j, r}^{(v)}(x, \rho)-e_{j}^{(v)}(x, \rho)\right) \exp (-i \rho x)\right|=0, \quad v=0,1 .
$$

Here $e_{j, r}(x, \rho)$ are the Jost solutions for the potentials $q_{j, r}(x)$.

Let $\Psi_{k}(\lambda)=\left[\Psi_{k j}(x, \lambda)\right]_{j=\overline{1, m}}, k=\overline{1, p}$, be solutions of (1), (2) satisfying the following boundary conditions in the boundary vertices:

$$
U_{j}\left(\Psi_{k j}(x, \lambda)\right)=-\delta_{j k}, \quad j=\overline{1, p},
$$

and having the following asymptotics on the noncompact edges:

$$
\Psi_{k j}(x, \lambda)=O(\exp (i \rho x)), \quad x \rightarrow \infty, \rho \in \overline{\Omega_{+}}, j=\overline{p+1, m} .
$$


The functions $\Psi_{k}(\lambda)$ and $M_{k}(\lambda):=\Psi_{k k}(1, \lambda)$ are called respectively the Weyl solution and the Weyl function associated with the boundary vertex $v_{k}, k=\overline{1, p}$.

We introduce also the solutions $f_{k}(\rho)=\left[f_{k j}(x, \rho)\right]_{j=\overline{1, m}}, k=\overline{p+1, m}$, of (1)-(3) having the following asymptotics on the noncompact edges:

$$
f_{k k}(x, \rho) \sim \exp (-i \rho x), \quad f_{k j}(x, \rho)=O(\exp (i \rho x)), \quad x \rightarrow \infty, \rho \in \Omega_{+}, j=\overline{p+1, m} \backslash k .
$$

The function $f_{k}(\rho)$ is called the scattering solution associated with the edge $\varepsilon_{k}, k=\overline{p+1, m}$.

In order to investigate the Weyl and scattering solutions we construct the auxiliary solutions $g_{k}(\rho)=\left[g_{k j}(x, \rho)\right]_{j=1, m}, k=\overline{1, m}$, of (1), (2) in the following way:

$$
\begin{aligned}
& g_{k j}(x, \rho)=e_{j}(x, \rho) \prod_{l \neq k, j} e_{l}(0, \rho), \quad j \neq k, \\
& g_{k k}(x, \rho)=C_{k}(x, \lambda) \prod_{l \neq k} e_{l}(0, \rho)-S_{k}(x, \lambda) \sum_{j \neq k} e_{j}^{\prime}(0, \rho) \prod_{l \neq k, j} e_{l}(0, \rho),
\end{aligned}
$$

where here and in the sequel for briefness we use the notation

$$
e_{j}(x, \rho):=\psi_{j}\left(x, \rho^{2}\right), \quad j=\overline{1, p} .
$$

Obviously $g_{k, j}(x, \rho)$ for each fixed $x \geq 0$ is analytic in $\Omega_{+}$and continuous in $\overline{\Omega_{+}}$. Moreover, we have

$$
\left.\begin{array}{cc}
U_{j}\left(g_{k j}(x, \rho)\right)=0, & j=\overline{1, p} \backslash k, \\
g_{k j}(x, \rho)=O(\exp (i \rho x)), & x \rightarrow \infty, \rho \in \overline{\Omega_{+}}, j=\overline{p+1, m} \backslash k .
\end{array}\right\}
$$

Lemma 2. The following representation holds

$$
\Psi_{k}(\lambda)=\frac{1}{\Delta(\lambda)} g_{k}(\rho), \quad k=\overline{1, p}
$$

where

$$
\Delta(\lambda)=\sum_{j=1}^{m} e_{j}^{\prime}(0, \rho) \prod_{l \neq j} e_{l}(0, \rho)
$$

Proof. According to (12), (13), (18) we have $\Psi_{k}(\lambda)=-\left(U_{k}\left(g_{k k}(x, \rho)\right)\right)^{-1} g_{k}(\rho), k=\overline{1, p}$. Using (3)-(5) we get

$$
U_{k}\left(C_{k}(x, \lambda)\right)=-\psi_{k}^{\prime}(0, \lambda)=-e_{k}^{\prime}(0, \rho), \quad U_{k}\left(S_{k}(x, \lambda)\right)=\psi_{k}(0, \lambda)=e_{k}(0, \rho), \quad k \in \overline{1, p},
$$

which together with (16) gives $U_{k}\left(g_{k k}(x, \rho)\right)=-\Delta(\lambda)$.

It is obvious that for $k=\overline{1, p}$ the following relations hold

$$
\Psi_{k k}(x, \lambda)=\theta_{k}(x, \lambda)+M_{k}(\lambda) \psi_{k}(x, \lambda)=\frac{g_{k k}(x, \rho)}{\Delta(\lambda)}, \quad M_{k}(\lambda)=\frac{g_{k k}(1, \rho)}{\Delta(\lambda)} .
$$


Thus, the Weyl solutions and the Weyl functions have a jump along the real semi-axis $[0, \infty)$ in the $\lambda$-plane.

Further, let $k=\overline{p+1, m}$. Then for $\rho \in \mathbb{R}^{*}$ we have

$$
g_{k k}(x, \rho)=a(\rho) e_{k}(x,-\rho)+b_{k}(\rho) e_{k}(x, \rho), \quad k=\overline{p+1, m},
$$

where $a(\rho)$ does not depend on $k$. Indeed, using (11) we have

$$
\left[\begin{array}{c}
a(\rho) \\
b_{k}(\rho)
\end{array}\right]=\frac{1}{2 i \rho}\left[\begin{array}{cc}
e_{k}^{\prime}(0, \rho) & -e_{k}(0, \rho) \\
-e_{k}^{\prime}(0,-\rho) & e_{k}(0,-\rho)
\end{array}\right]\left[\begin{array}{l}
g_{k k}(0, \rho) \\
g_{k k}^{\prime}(0, \rho)
\end{array}\right] .
$$

Thus, using (15), (16), (20) we calculate

$$
\begin{aligned}
a(\rho) & =\frac{1}{2 i \rho}\left\langle g_{k k}(x, \rho), e_{k}(x, \rho)\right\rangle=\frac{1}{2 i \rho} \Delta\left(\rho^{2}\right), \\
b_{k}(\rho) & =-\frac{1}{2 i \rho}\left(e_{k}^{\prime}(0,-\rho) \prod_{l \neq k} e_{l}(0, \rho)+e_{k}(0,-\rho) \sum_{j \neq k} e_{j}^{\prime}(0, \rho) \prod_{l \neq k, j} e_{l}(0, \rho)\right) .
\end{aligned}
$$

The second equality in (23) is valid for $k=\overline{1, m}$.

We note that (23) gives an analytic continuation of $a(\rho)$ in $\Omega_{+}$. Hence the function $a(\rho)$ is analytic in $\Omega_{+}$and $\rho a(\rho)$ is continuous in $\overline{\Omega_{+}}$. Moreover, using (20), (23) together with the asymptotics of the Jost solutions (10) and the entire solutions $e_{j}(x, \rho)=\psi_{j}\left(x, \rho^{2}\right), j=\overline{1, p}$, (6) for each $\varepsilon>0$ we get

$$
a(\rho)=\frac{m}{2^{p+1}} \exp (-p i \rho)\left(1+\frac{1}{i \rho} \sum_{j=1}^{m} \omega_{j}(0)+o\left(\frac{1}{\rho}\right)\right), \quad|\rho| \rightarrow \infty, \quad \rho \in \Omega_{\varepsilon},
$$

where $\Omega_{\varepsilon}=\{\rho: \arg \rho \in[\varepsilon, \pi-\varepsilon]\}$.

Lemma 3. For $\rho \in \mathbb{R}^{*}, k=\overline{p+1, m}$ the following relations hold:

$$
\begin{aligned}
\overline{a(\rho)} & =a(-\rho), \quad \overline{b_{k}(\rho)}=b_{k}(-\rho), \\
|a(\rho)|^{2}-\left|b_{k}(\rho)\right|^{2} & =\sum_{\substack{j \neq k \\
j=p+1}}^{m} \prod_{l \neq k, j}\left|e_{l}(0, \rho)\right|^{2} .
\end{aligned}
$$

Proof. By virtue of (7), (17) we have $e_{j}(x,-\rho)=e_{j}(x, \rho)=\overline{e_{j}(x, \rho)}$ for $j=\overline{1, p}$ and $e_{j}(x,-\rho)=$ $\overline{e_{j}(x, \rho)}$ for $j=\overline{p+1, m}, \rho \in \mathbb{R}$. Thus, (26) follows from (23), (24). Further, (11), (22) give

$$
\left\langle g_{k k}(x, \rho), g_{k k}(x,-\rho)\right\rangle=2 i \rho\left(a(\rho) a(-\rho)-b_{k}(\rho) b_{k}(-\rho)\right), \quad k=\overline{p+1, m} .
$$

On the other hand, according to (11), (16) we have

$$
\left\langle g_{k k}(x, \rho), g_{k k}(x,-\rho)\right\rangle=2 i \rho \sum_{\substack{j \neq k \\ j=p+1}}^{m} \prod_{l \neq k, j} e_{l}(0, \rho) e_{l}(0,-\rho), \quad k=\overline{p+1, m},
$$

which together with (26), (28) give (27). 
Corollary 1. Let $\rho \in \mathbb{R}^{*}$. Since $e_{j}(x, \rho) \neq 0, x \geq 0, j=\overline{p+1, m}$, according to (20), (23), (27) we have $a(\rho)=0$ only if $\psi_{l_{v}}\left(0, \rho^{2}\right)=0$ for at least two distinct indices $l_{v} \in\{1, \ldots, p\}, v=1,2$. Moreover, for all pairs of distinct indices $k, j \in\{p+1, \ldots, m\}$ we have

$$
\left|\frac{1}{a(\rho)} \prod_{l \neq k, j} e_{l}(0, \rho)\right| \leq 1, \quad \rho \in \mathbb{R}_{1}^{*}:=\left\{\rho: \rho \in \mathbb{R}^{*}, a(\rho) \neq 0\right\} .
$$

Lemma 4. For $\rho \in \Omega_{+}$such that $a(\rho) \neq 0$ the following representation holds:

$$
f_{k}(\rho)=\frac{1}{a(\rho)} g_{k}(\rho), \quad k=\overline{p+1, m}
$$

Proof. According to (8), (14) we have

$$
\left\langle e_{k}(x, \rho), f_{k k}(x, \rho)\right\rangle=-2 i \rho .
$$

Let us first show that if $a(\rho) \neq 0$ then

$$
f_{k}(\rho)=D_{k}(\rho) g_{k}(\rho)
$$

For $a(\rho) \neq 0$ and $j \neq k$ it is easy to show that $f_{k j}(x, \rho)=D_{k j}(\rho) g_{k j}(x, \rho)$, whence according to (2), (15) we get

$$
\left(D_{k j_{1}}(\rho)-D_{k j_{2}}(\rho)\right) \prod_{j \neq k} e_{j}(0, \rho)=0, \quad j_{1}, j_{2} \neq k
$$

Thus, if

$$
\prod_{j \neq k} e_{j}(0, \rho) \neq 0,
$$

then all $D_{k j}(\rho), j \neq k$, are equal and we put $D_{k}(\rho):=D_{k j}(\rho)$. Let (33) be false. Then there exists a unique $j_{0} \neq k$ such that $e_{j_{0}}(0, \rho)=0$ (otherwise (20), (23) infer $a(\rho)=0$ ). Taking (15) into account we obtain $g_{k j}(x, \rho) \equiv 0$, for $j \neq k$, $j_{0}$ and put $D_{k}(\rho)=D_{k j_{0}}(\rho)$. Thus, we have $f_{k j}(x, \rho)=D_{k}(\rho) g_{k j}(x, \rho), j \neq k$. Since both the functions $f_{k}(\rho), g_{k}(\rho)$ satisfy the matching conditions (2) we get

$$
f_{k k}(x, \rho)=D_{k}(\rho) g_{k k}(x, \rho)
$$

and arrive at (32). Substituting (34) into (31) and using (23) we obtain

$$
-2 i \rho=D_{k}(\rho)\left\langle e_{k}(x, \rho), g_{k k}(x, \rho)\right\rangle=-2 i \rho D_{k}(\rho) a(\rho),
$$

which together with (32) gives (30).

For $\rho \in \mathbb{R}_{1}^{*}$ we consider (30) as a definition of the function $f_{k}(\rho)$. By virtue of the continuity of the functions $e_{k}(x, \rho), f_{k k}(x, \rho)$ with respect to $\rho$ in their domains of definition, formula (31) remains valid also for $\rho \in \mathbb{R}_{1}^{*}$. 


\section{Spectral-scattering data}

According to (22) and (30) we get

$$
f_{k k}(x, \rho)=e_{k}(x,-\rho)+s_{k}(\rho) e_{k}(x, \rho), \quad \rho \in \mathbb{R}_{1}^{*}, \quad k=\overline{p+1, m},
$$

where the function

$$
s_{k}(\rho)=\frac{b_{k}(\rho)}{a(\rho)}
$$

is called the reflection coefficient associated with the edge $\varepsilon_{k}, k=\overline{p+1, m}$.

Let us point out some properties of $s_{k}(\rho)$. By virtue of (23), (24), (26), (36) the functions $s_{k}(\rho)$ are continuous for $\rho \in \mathbb{R}_{1}^{*}$, and

$$
\overline{s_{k}(\rho)}=s_{k}(-\rho)
$$

Moreover, (27) implies

$$
\left|s_{k}(\rho)\right|^{2}=1-\frac{1}{|a(\rho)|^{2}} \sum_{\substack{j \neq k \\ j=p+1}}^{m} \prod_{l \neq k, j}\left|e_{l}(0, \rho)\right|^{2},
$$

and consequently we have

$$
\left|s_{k}(\rho)\right|<1, \quad \rho \in \mathbb{R}_{1}^{*} .
$$

Let us now study the properties of the discrete spectrum.

Definition 1. The values of the parameter $\lambda$, for which equation (1) has nonzero solutions $y=$ $\left[y_{j}(x)\right]_{j=\overline{1, m}}$, satisfying the matching conditions (2), the boundary conditions (3) and $y_{j}(x) \in$ $L_{2}(0, \infty), j=\overline{p+1, m}$, are called eigenvalues of $L$, and the corresponding nonzero solutions are called eigenfunctions.

Denote by $\Lambda$ the set of all eigenvalues of $L$ and put

$$
\begin{aligned}
\Lambda_{1} & :=\left\{\lambda=\rho^{2}: \rho \in \Omega_{+}, a(\rho)=0\right\}, \quad \Lambda_{2}:=\left\{\lambda=\rho^{2}: \rho \in \mathbb{R}^{*}, a(\rho)=0\right\}, \\
\Delta_{0}(\lambda) & :=\sum_{j=1}^{p} \psi_{j}^{\prime}(0, \lambda) \prod_{\substack{l \neq j \\
l=1}}^{p} \psi_{l}(0, \lambda), \\
\Delta_{1}(\lambda) & :=\prod_{j=1}^{p} \psi_{j}(0, \lambda), \\
\Lambda_{3} & :=\left\{\lambda: \lambda \geq 0, \Delta_{0}(\lambda)=\Delta_{1}(\lambda)=0\right\} .
\end{aligned}
$$

According to (20), (23) we have

$$
a(\rho)=\Delta_{0}\left(\rho^{2}\right) a_{1}(\rho)+\Delta_{1}\left(\rho^{2}\right) a_{0}(\rho),
$$


where

$$
a_{0}(\rho):=\frac{1}{2 i \rho} \sum_{j=p+1}^{m} e_{j}^{\prime}(0, \rho) \prod_{\substack{l \neq j \\ l=p+1}}^{m} e_{l}(0, \rho), \quad a_{1}(\rho):=\frac{1}{2 i \rho} \prod_{j=p+1}^{m} e_{j}(0, \rho) .
$$

Consider the boundary value problem $L_{0}$ for equation (1) on the graph $\Gamma_{0}$ that is obtained from $\Gamma$ by removing the noncompact edges $\varepsilon_{j}, j=\overline{p+1, m}$, with the new matching conditions

$$
y_{1}(0)=\ldots=y_{p}(0), \quad y_{1}^{\prime}(0)+\ldots+y_{p}^{\prime}(0)=0
$$

and the boundary conditions (3). It is obvious that the eigenvalues of $L_{0}$ coincide with the zeros of the entire function $\Delta_{0}(\lambda)$.

Lemma 5. $\Lambda_{2}=\Lambda_{3} \backslash\{0\}$.

Proof. According to (38) it is sufficient to prove the inclusion $\Lambda_{2} \subset \Lambda_{3}$. Let $\lambda=\rho^{2} \in \Lambda_{2}$, then (26), (38) give

$$
\left\{\begin{array}{c}
\Delta_{0}(\lambda) a_{1}(\rho)+\Delta_{1}(\lambda) a_{0}(\rho)=0 \\
\Delta_{0}(\lambda) a_{1}(-\rho)+\Delta_{1}(\lambda) a_{0}(-\rho)=0 .
\end{array}\right.
$$

By virtue of (11), (39) the determinant of this linear algebraic system does not vanish:

$$
a_{1}(\rho) a_{0}(-\rho)-a_{1}(-\rho) a_{0}(\rho)=\frac{1}{2 i \rho} \sum_{j=p+1}^{m} \prod_{\substack{l \neq j \\ l=p+1}}^{m}\left|e_{l}(0, \rho)\right|^{2} \neq 0 .
$$

Thus, from (40) it follows that $\lambda \in \Lambda_{3}$.

Theorem 2. The set $\Lambda=\left\{\lambda_{n}\right\}$ is at most countable. All eigenvalues $\lambda_{n}$ are real. Eigenfunctions related to different eigenvalues are orthogonal in $L_{2}(\Gamma)$. For each eigenvalue there exist up to $m-1$ linearly independent eigenfunctions. Moreover,

(i) $\Lambda=\Lambda_{1} \cup \Lambda_{3}$;

(ii) $y_{j}(x) \equiv 0, j=\overline{p+1, m}$, for each eigenfunction $y=\left[y_{j}(x)\right]_{j=1, m}$ related to $\lambda \in \Lambda_{3}$.

(iii) for all $\lambda_{n}=\rho_{n}^{2} \in \Lambda$

$$
g_{k k}\left(x, \rho_{n}\right)=d_{k n} e_{k}\left(x, \rho_{n}\right), \quad k=\overline{1, m} .
$$

(iv) $g_{k}\left(\rho_{n}\right)$ is an eigenfunction related to $\lambda_{n}$ if and only if $d_{k n} \neq 0$.

Proof. Since $\mathscr{L}$ is a selfadjoint operator in $L_{2}(\Gamma)$, it is known [42] that its eigenvalues are real and eigenfunctions related to different eigenvalues are orthogonal in $L_{2}(\Gamma)$.

The components $y_{j}(x)$ of each eigenfunction $y=\left[y_{j}(x)\right]_{j=\overline{1, m}}$ corresponding to an eigenvalue $\lambda=\rho^{2}, \rho \in \Omega_{+}$, have the form $y_{j}(x)=A_{j} e_{j}(x, \rho)$, where $A_{j}$ are constants, which do not 
vanish simultaneously. Substituting this into the matching conditions (2) we arrive at a homogeneous system of linear algebraic equations with respect to $A_{j}$, whose determinant equals to $\Delta\left(\rho^{2}\right)$. Thus, the set of eigenvalues $\lambda=\rho^{2}, \rho \in \Omega_{+}$, coincides with $\Lambda_{1}$. Moreover, since the rank of the determinant $\Delta(\lambda)$ exceeds 0 , the number of linearly independent eigenfunctions related to an eigenvalue from $\Lambda_{1}$ is not greater than $m-1$.

Further, let $\lambda \geq 0$ be an eigenvalue and let $y=\left[y_{j}(x)\right]_{j=1, m}$ be a corresponding eigenfunction. Using the same arguments as in [24] one can show that $y_{j}(x) \equiv 0, j=\overline{p+1, m}$. Thus, $y^{0}:=\left[y_{j}(x)\right]_{j=\overline{1, p}}$ is an eigenfunction of the boundary value problem $L_{0}$, and hence $\lambda \in \Lambda_{3}$. Conversely, each $\lambda \in \Lambda_{3}$ is an eigenvalue of $L_{0}$ and the corresponding eigenfunctions vanish in $v_{0}$, hence it is an eigenvalue also of $L$. Thus, the set of real nonnegative eigenvalues coincides with $\Lambda_{3}$ and (i), (ii) are proved. Moreover, it is obvious that the number of linearly independent eigenfunctions related to each $\lambda \in \Lambda_{3}$ does not exceed $p-1$.

For $\lambda_{n} \in \Lambda$ according to (23), (38), (39) and the first assertion (i) of the theorem we have $\Delta\left(\lambda_{n}\right)=0$. Using the second equality in (23) for all $k=\overline{1, m}$ we obtain (41). Further, let $d_{k n} \neq 0$. Then for $\lambda_{n} \in \Lambda_{1}$ it is obvious that $g_{k}\left(\rho_{n}\right)$ is an eigenfunction. If $\lambda_{n}=\rho_{n}^{2} \in \Lambda_{3}$ we have $e_{j}\left(x, \rho_{n}\right) \notin L_{2}(0, \infty), j=\overline{p+1, m}$. However, for $k=\overline{1, p}$ the function $g_{k}\left(\rho_{n}\right)$ is an eigenfunction anyway because in this case according to (15), (37) we have $g_{k j}(x, \rho) \equiv 0$ for $j=\overline{p+1, m}$. Note that according to (16) for $k=\overline{p+1, m}$ and $\lambda_{n} \in \Lambda_{3}$ we have $d_{k n}=0$. Conversely, let $d_{k n}=0$. Then $g_{k k}\left(x, \rho_{n}\right) \equiv 0$ and according to (16) there are two distinct indices $j_{1}, j_{2} \neq k$ such that $e_{j_{v}}\left(0, \rho_{n}\right)=0, v=1,2$. By virtue of (15) we get $g_{k}\left(\rho_{n}\right)=0$, and hence $g_{k}\left(\rho_{n}\right)$ is not an eigenfunction.

Theorem 3. The set of negative eigenvalues $\Lambda_{1}$ is finite.

Proof. According to (25) $\Lambda_{1} \subset(-M, 0), M<\infty$. Hence, the set of negative eigenvalues $\lambda_{n}=$ $\rho_{n}^{2}, \operatorname{Im} \rho_{n}>0$, has a uniquely possible accumulation point in the origin. Let us assume to the contrary that $\Lambda_{1}$ is infinite, then $\tau_{n}:=-i \rho_{n} \rightarrow 0, n \rightarrow \infty$. Since $e_{j}\left(0, \rho_{n}\right) \neq 0, j=\overline{1, m}$, for sufficiently large $n$ (for $j=\overline{p+1, m}$ see, e.g., [27]), we have $g_{k}\left(\rho_{n}\right) \neq 0, k=\overline{1, m}$, for large $n$, and hence $g_{k}\left(\rho_{n}\right)$ is an eigenfunction. According to (7), (16), (41) we get $d_{k n} \in \mathbb{R}$. One can choose such increasing sequences of natural numbers $\left\{n_{v}\right\},\left\{n_{v}^{\prime}\right\}$ that for all $v \in \mathbb{N}$

$$
n_{v} \neq n_{v}^{\prime}, \quad d_{1 n_{v}} d_{1 n_{v}^{\prime}}>0, \quad e_{j}\left(0, \rho_{n_{v}}\right) e_{j}\left(0, \rho_{n_{v}^{\prime}}\right)>0, \quad j=\overline{1, m} .
$$

By virtue of (9), there exists $A>0$ such that

$$
\int_{A}^{\infty} e_{j}\left(x, i \tau^{\prime}\right) e_{j}\left(x, i \tau^{\prime \prime}\right) d x \geq \frac{\exp (-2 A M)}{8 M}, \quad \tau^{\prime}, \tau^{\prime \prime} \in(0, M], j=\overline{p+1, m} .
$$

Hence for sufficiently large $v$ we obtain

$$
\int_{0}^{\infty} e_{j}\left(x, \rho_{n_{v}}\right) e_{j}\left(x, \rho_{n_{v}^{\prime}}\right) d x=\int_{A}^{\infty} e_{j}\left(x, \rho_{n_{v}}\right) e_{j}\left(x, \rho_{n_{v}^{\prime}}\right) d x
$$




$$
\begin{gathered}
\quad+\int_{0}^{A} e_{j}\left(x, \rho_{n_{v}}\right)\left(e_{j}\left(x, \rho_{n_{v}^{\prime}}\right)-e_{j}\left(x, \rho_{n_{v}}\right)\right) d x+\int_{0}^{A} e_{j}^{2}\left(x, \rho_{n_{v}}\right) d x>0, \quad j=\overline{p+1, m}, \\
\int_{0}^{1} e_{j}\left(x, \rho_{n_{v}}\right) e_{j}\left(x, \rho_{n_{v}^{\prime}}\right) d x=\int_{0}^{1} \psi_{j}\left(x, \lambda_{n_{v}}\right) \psi_{j}\left(x, \lambda_{n_{v}^{\prime}}\right) d x \\
=\int_{0}^{1} \psi_{j}\left(x, \lambda_{n_{v}}\right)\left(\psi_{j}\left(x, \lambda_{n_{v}^{\prime}}\right)-\psi_{j}\left(x, \lambda_{n_{v}}\right) d x+\int_{0}^{1} \psi_{j}^{2}\left(x, \lambda_{n_{v}}\right) d x>0, \quad j=\overline{1, p} .\right.
\end{gathered}
$$

Take, for example, $g_{p+1}(\rho)$. According to (15), (41)-(44) we have

$$
\begin{aligned}
\sum_{j=1}^{m} \int_{0}^{\infty} g_{p+1, j}\left(x, \rho_{n_{v}}\right) g_{p+1, j}\left(x, \rho_{n_{v}^{\prime}}\right) d x \\
=d_{p+1, n_{v}} d_{p+1, n_{v}^{\prime}} \int_{0}^{\infty} e_{p+1}\left(x, \rho_{n_{v}}\right) e_{p+1}\left(x, \rho_{n_{v}^{\prime}}\right) d x \\
\quad+\sum_{\substack{j \neq p+1 \\
j=1}}^{m} \prod_{\substack{l \neq p+1, j \\
e_{l}}}\left(0, \rho_{n_{v}}\right) e_{l}\left(0, \rho_{n_{v}^{\prime}}\right) \int_{0}^{\infty} e_{j}\left(x, \rho_{n_{v}}\right) e_{j}\left(x, \rho_{n_{v}^{\prime}}\right) d x>0,
\end{aligned}
$$

where $g_{p+1, j}(x, \rho)=0$ and $e_{j}(x, \rho)=0$ for $x>1, j=\overline{1, p}$. Inequality (45) contradicts the orthogonality of the eigenfunctions $g_{p+1}\left(\rho_{n_{v}}\right), g_{p+1}\left(\rho_{n_{v}^{\prime}}\right)$ related to the different eigenvalues $\lambda_{n_{v}}, \lambda_{n_{v}^{\prime}}$

Thus, the set of negative eigenvalues has the form

$$
\Lambda_{1}=\left\{\lambda_{n}\right\}_{n=\overline{1, N}}, \quad \lambda_{n}=\rho_{n}^{2}, \rho_{n}=i \tau_{n}, 0<\tau_{N}<\ldots<\tau_{1} .
$$

For briefness denote

$$
f_{k}(\rho):=\Psi_{k}\left(\rho^{2}\right), \quad f_{k j}(x, \rho):=\psi_{k j}\left(x, \rho^{2}\right), \quad j=\overline{1, m}, \quad k=\overline{1, p} .
$$

Theorem 4. The functions $f_{k}(\rho), k=\overline{1, m}$, have at most simple poles in $\rho_{n}, n=\overline{1, N}$. The corresponding nonzero residues are eigenfunctions related to the eigenvalues $\lambda_{n}=\rho_{n}^{2}$.

Proof. Let $\rho_{n}$ be a pole of order $s \geq 1$ of the function $f_{k}(\rho)$. According to (3), (5), (8), (12), (15), (17), (19), (30), (31), (46) we have

$$
\left\langle f_{k j}(x, \rho), e_{j}(x, \rho)\right\rangle=\delta_{k j}\left\{\begin{aligned}
1, k & =\overline{1, p}, \\
2 i \rho, k & =\overline{p+1, m}, \quad j=\overline{1, m},
\end{aligned}\right.
$$

where $\delta_{k j}$ is the Kronecker delta. Consider the function $u=\left[u_{j}(x)\right]_{j=\overline{1, m}}$, determined by the formula

$$
u=\lim _{\rho \rightarrow \rho_{n}}\left(\rho-\rho_{n}\right)^{s} f_{k}(\rho)
$$

Formulae (47), (48) give

$$
\left\langle u_{j}(x), e_{j}\left(x, \rho_{n}\right)\right\rangle=0, \quad j=\overline{1, m},
$$


and consequently

$$
-u_{j}^{\prime \prime}+q_{j}(x) u_{j}=\lambda_{n} u_{j}, \quad x \in \varepsilon_{j}, \quad j=\overline{1, m} ; \quad U_{j}\left(u_{j}\right)=0, j=\overline{1, p} .
$$

Since the function $f_{k}(\rho)$ satisfies the matching conditions (2), the function $u$ satisfies them too. Thus, $u$ is an eigenfunction corresponding to the eigenvalue $\lambda_{n}$.

Let us assume to the contrary that $s>1$. Determine the function $v=\left[v_{j}(x)\right]_{j=\overline{1, m}}$ by the formula

$$
v=\lim _{\rho \rightarrow \rho_{n}} \frac{d}{d \rho}\left(\left(\rho-\rho_{n}\right)^{s} f_{k}(\rho)\right) .
$$

By virtue of (47), (48) we have

$$
\left\langle v_{j}(x), e_{j}\left(x, \rho_{n}\right)\right\rangle+\left\langle u_{j}(x), \dot{e}_{j}\left(x, \rho_{n}\right)\right\rangle=0, \quad j=\overline{1, m},
$$

where $\dot{e}_{j}(x, \rho)=\frac{d}{d \rho} e_{j}(x, \rho)$. Differentiating (51) with respect to $x$ and then substituting

$$
e_{j}^{\prime \prime}\left(x, \rho_{n}\right)=\left(q_{j}(x)-\rho_{n}^{2}\right) e_{j}\left(x, \rho_{n}\right), \quad \dot{e}_{j}^{\prime \prime}\left(x, \rho_{n}\right)=\left(q_{j}(x)-\rho_{n}^{2}\right) \dot{e}_{j}\left(x, \rho_{n}\right)-2 \rho_{n} e_{j}\left(x, \rho_{n}\right)
$$

and (50) into the relation obtained, we arrive at

$$
-v_{j}^{\prime \prime}(x)+q_{j}(x) v_{j}(x)=\rho_{n}^{2} v_{j}(x)+2 \rho_{n} u_{j}(x), \quad x \in \varepsilon_{j}, \quad j=\overline{1, m} .
$$

Using (50), (52) we obtain

$$
\left.\sum_{j=1}^{p}\left\langle v_{j}(x), u_{j}(x)\right\rangle\right|_{x=0} ^{1}+\left.\sum_{j=p+1}^{m}\left\langle v_{j}(x), u_{j}(x)\right\rangle\right|_{x=0} ^{\infty}=2 \rho_{n}\left(\sum_{j=1}^{p} \int_{0}^{1} u_{j}^{2}(x) d x+\sum_{j=p+1}^{m} \int_{0}^{\infty} u_{j}^{2}(x) d x\right) .
$$

Since the functions $u, v$ satisfy the matching conditions (2), we get

$$
\left.\sum_{j=1}^{m}\left\langle v_{j}(x), u_{j}(x)\right\rangle\right|_{x=0}=0 \text {. }
$$

From (49) we get $u_{j}(x)=C_{j} e_{j}\left(x, \rho_{n}\right), j=\overline{1, m}$, which together with (3), (5), (17) gives

$$
\left.\left\langle v_{j}(x), u_{j}(x)\right\rangle\right|_{x=1}=-C_{j} U_{j}\left(v_{j}(x)\right)=0, \quad j=\overline{1, p} .
$$

Moreover, for $j=\overline{p+1, m}$ we have $\dot{e}_{j}^{(v)}\left(x, \rho_{n}\right)=O(1), x \rightarrow \infty, v=0,1$, (see [31]). By virtue of (51) we have

$$
\begin{aligned}
\lim _{x \rightarrow \infty}\left\langle v_{j}(x), u_{j}(x)\right\rangle & =C_{j} \lim _{x \rightarrow \infty}\left\langle v_{j}(x), e_{j}\left(x, \rho_{n}\right)\right\rangle= \\
& =C_{j} \lim _{x \rightarrow \infty}\left\langle\dot{e}_{j}\left(x, \rho_{n}\right), u_{j}(x)\right\rangle=0, j=\overline{p+1, m},
\end{aligned}
$$

because $u_{j}^{(v)}(x)=o(1), x \rightarrow \infty, v=0,1$. According to (7), (15), (16), (23), (30) the functions $f_{k}(i \tau), \tau \geq 0$, are real-valued. Thus, by virtue of (48) the function $u(x)$ is real or imaginary depending on the fact if $s$ is even or odd, respectively. Taking into account (53)-(56) we get $u(x) \equiv 0$, which is impossible. 
Corollary 2. For the eigenvalue $\lambda_{n_{0}}=\rho_{n_{0}}^{2} \in \Lambda_{1}$ to be multiple, i.e. $a\left(\rho_{n_{0}}\right)=\dot{a}\left(\rho_{n_{0}}\right)=0$, it is necessary and sufficient that there are at least three distinct numbers $j_{1}, j_{2}, j_{3} \in\{1, \ldots, m\}$, such that $e_{j_{v}}\left(0, \rho_{n_{0}}\right)=0, v=\overline{1,3}$.

Proof. The sufficiency follows from (20), (23). Let $a\left(\rho_{n_{0}}\right)=\dot{a}\left(\rho_{n_{0}}\right)=0$, then according to Theorem 4 and formula (30) we have $g_{k j}\left(x, \rho_{n_{0}}\right) \equiv 0$ for all $k, j \in\{1, \ldots, m\}$. Hence, for all $k, j$ in the right-hand part of (15) for $\rho=\rho_{n_{0}}$ there should be at least one zero-multiplier $e_{l}\left(0, \rho_{n_{0}}\right)=$ $0, l \neq k, j$, which proves the necessity.

Lemma 6. For all $k, j \in\{p+1, \ldots, m\}$ and $\mu$ such that $\mu^{2} \in \Lambda_{3} \cup\{0\}$ the following estimate holds:

$$
a_{k j}(\rho):=\frac{1}{a(\rho)} \prod_{l \neq k, j} e_{l}(0, \rho)=O(1), \quad \rho \rightarrow \mu, \rho \in \overline{\Omega_{+}} .
$$

Proof. Fix $k, j \in\{p+1, \ldots, m\}$ and $\mu$ such that $\mu^{2} \in \Lambda_{3} \cup\{0\}$. Suppose that the functions $e_{l}^{(v)}(0, \rho), l=\overline{p+1, m}, v=0,1$, are analytic in a vicinity of $\mu$. Then, using (20), (23), (29) we deduce that the function $a_{k j}(\rho)$ has a removable singularity in $\mu$, and hence (57) holds.

In the general case we cannot use these arguments. Therefore, we introduce the potential $q_{(r)}=\left[q_{j, r}(x)\right]_{j=\overline{1, m}}$, where

$$
q_{j, r}(x)=q_{j}(x), \quad j=\overline{1, p}, \quad q_{j, r}(x)=\left\{\begin{array}{c}
q_{j}(x), 0<x<r, \\
0, \quad x>r,
\end{array} \quad j=\overline{p+1, m},\right.
$$

and consider the corresponding Jost solutions $e_{j, r}(x, \rho), j=\overline{p+1, m}$. Clearly, for each fixed $x$ the functions $e_{j, r}^{(v)}(x, \rho), v=0,1$, are entire in $\rho$. Put

$$
a_{r}(\rho)=\frac{1}{2 i \rho} \sum_{j=1}^{m} e_{j, r}^{\prime}(0, \rho) \prod_{l \neq j} e_{l, r}(0, \rho),
$$

where $e_{j, r}(x, \rho)=\psi_{j}\left(x, \rho^{2}\right), j=\overline{1, p}$. By virtue of Lemma 1 and (6), (10) for each fixed $\varepsilon>0$ we have

$$
\lim _{r \rightarrow \infty} a_{r}(\rho) \exp (\text { pi } \rho)=a(\rho) \exp (\text { pi } \rho) \text { uniformly in } \overline{\Omega_{+}} \backslash\{\rho:|\rho|<\varepsilon\} .
$$

Choose $\delta>0$ such that $a(\rho) \neq 0$ for $\rho \in[\mu-\delta, \mu) \cup(\mu, \mu+\delta]$ and consider the set $O_{\delta}(\mu):=\{\rho$ : $0<|\rho-\mu| \leq \delta\} \cap \overline{\Omega_{+}}$. Denote

$$
a_{k j, r}(\rho):=\frac{1}{a_{r}(\rho)} \prod_{l \neq k, j} e_{l, r}(0, \rho) .
$$

(i) Let $\mu \neq 0$, then according to (58)

$$
\lim _{r \rightarrow \infty} a_{k j, r}(\rho)=a_{k j}(\rho)
$$


uniformly with respect to $\rho \in O_{\delta}(\mu) \backslash O_{\varepsilon}(\mu)$ for each fixed $\varepsilon>0$. This means, in particular, that $\left|a_{k j, r}(\rho)\right| \leq C,|\rho|=\delta$, where $C$ does not depend on $r$. Moreover, according to Corollary 1 we have $\left|a_{k j, r}(\rho)\right| \leq 1$ for $\rho \in \mathbb{R}$. Using the maximum modulus principle we get

$$
\left|a_{k j, r}(\rho)\right| \leq C, \quad \rho \in O_{\delta}(\mu)
$$

Taking in (59) the limit as $r \rightarrow \infty$ we arrive at $\left|a_{k j}(\rho)\right| \leq C, \rho \in O_{\delta}(\mu)$. Thus, (57) is proved for $\mu \neq 0$.

(ii) Let $\mu=0$. According to Lemma 5 and Theorem $3 \rho=0$ is not a concentration point for the set $\left\{\rho: \rho \in \overline{\Omega_{+}} \backslash\{0\}, a(\rho)=0\right\}$. Hence, there exists $\delta>0$ such that $a(\rho) \neq 0$ for all $\rho \in O_{\delta}(0)$. By virtue of (58) for each $\varepsilon>0$ there exists $R$ such that $a_{r}(\rho) \neq 0$ for $\rho \in O_{\delta}(0) \backslash O_{\varepsilon}(0), r \geq R$. Let $\rho_{n, r}, n=\overline{1, N_{r}}$, be all zeros of the function $a_{r}(\rho)$ in $O_{\varepsilon}$ counted with multiplicity.

Let us show that $N_{r} \leq K$ for all $r$. Since the function $e_{j, r}(0, \rho)$ has a uniformly bounded number of zeros (see [27]), it is sufficient to prove that the number of the zeros $\rho_{n, r}$ of $a_{r}(\rho)$, such that $e_{j, r}\left(0, \rho_{n, r}\right) \neq 0, j=\overline{1, m}$, is uniformly bounded too. Construct the solution $g_{k, r}(\rho)=$ $\left[g_{k j, r}(x, \rho)\right]_{j=\overline{1, m}}$ for the potential $q_{(r)}$ analogously to $g_{k}(\rho)$ for $q$. According to Theorem 2 and (16) $g_{k, r}\left(\rho_{n, r}\right)$ is an eigenfunction of $L\left(q_{(r)}, H\right)$ related to the eigenvalue $\rho_{n, r}^{2}$ and

$$
g_{k k, r}\left(x, \rho_{n, r}\right)=d_{k n, r} e_{k, r}\left(x, \rho_{n, r}\right), \quad d_{k n, r} \neq 0, \quad k=\overline{1, m} .
$$

Assume to the contrary that the number of zeros $N_{r}$ is not bounded. Then there exist such increasing sequences $\left\{r_{v}\right\},\left\{n_{v}\right\},\left\{n_{v}^{\prime}\right\}$ that

$$
n_{v} \neq n_{v}^{\prime}, \quad d_{1 n_{v}, r_{v}} d_{1 n_{v}^{\prime}, r_{v}}>0, \quad e_{j, r_{v}}\left(0, \rho_{n_{v}, r_{v}}\right) e_{j, r_{v}}\left(0, \rho_{n_{v}^{\prime}, r_{v}}\right)>0, \quad j=\overline{1, m}
$$

Analogously to the estimates (43), (44) we obtain

$$
\int_{0}^{\infty} e_{j, r_{v}}\left(x, \rho_{n_{v}, r_{v}}\right) e_{j, r_{v}}\left(x, \rho_{n_{v}^{\prime}, r_{v}}\right) d x>0, \quad j=\overline{1, m}
$$

for sufficiently large $v$. According to (15), (60) and (61), (62) we have

$$
\begin{aligned}
\sum_{j=1}^{m} \int_{0}^{\infty} g_{1 j, r_{v}}\left(x, \rho_{n_{v}, r_{v}}\right) g_{1 j, r_{v}}\left(x, \rho_{n_{v}^{\prime}, r_{v}}\right) d x \\
=d_{1 n_{v}, r_{v}} d_{1 n_{v}^{\prime}, r_{v}} \int_{0}^{\infty} e_{1, r_{v}}\left(x, \rho_{n_{v}, r_{v}}\right) e_{1, r_{v}}\left(x, \rho_{n_{v}^{\prime}, r_{v}}\right) d x \\
\quad+\sum_{j=2}^{m} \prod_{l \neq j} e_{l, r_{v}}\left(0, \rho_{n_{v}, r_{v}}\right) e_{l, r_{v}}\left(0, \rho_{n_{v}^{\prime}, r_{v}}\right) \int_{0}^{\infty} e_{j, r_{v}}\left(x, \rho_{n_{v}, r_{v}}\right) e_{j, r_{v}}\left(x, \rho_{n_{v}^{\prime}, r_{v}}\right) d x>0,
\end{aligned}
$$

which contradicts the orthogonality in $L_{2}(\Gamma)$ of the eigenfunctions $g_{1, r_{v}}\left(\rho_{n_{v}, r_{v}}\right), g_{1, r_{v}}\left(\rho_{n_{v}^{\prime}, r_{v}}\right)$. Thus, we have proved that $N_{r} \leq K$. 
Further, put

$$
A_{k j, r}(\rho):=a_{k j, r}(\rho) \varphi_{r}(\rho), \quad \varphi_{r}(\rho)=\prod_{n=1}^{N_{r}} \frac{\rho-\rho_{n, r}}{\rho+\rho_{n, r}} .
$$

After removing its singularities the function $A_{k j, r}(\rho)$ is analytic in $O_{\delta}(0)$. It is obvious that $\left|\varphi_{r}(\rho)\right| \leq 1, \rho \in \overline{\Omega_{+}}$, and

$$
\lim _{r \rightarrow \infty} \varphi_{r}(\rho)=1, \quad \rho \neq 0 .
$$

By virtue of (58), (63), (64) and Lemma 1 we obtain

$$
\lim _{r \rightarrow \infty} A_{k j, r}(\rho)=a_{k j}(\rho), \quad \rho \in O_{\delta}(0) .
$$

Using (59), (63) we get $\left|a_{k j}(\rho)\right| \leq C, \rho \in O_{\delta}(0)$. Thus, (57) is proved also for $\mu=0$.

Corollary 3. For all fixed $x \geq 0, k=\overline{p+1, m}, v=0,1$ and $\mu$ such that $\mu^{2} \in \Lambda_{2}$ the following estimates hold:

$$
f_{k k}^{(v)}(x, \rho)=O\left(\frac{1}{\rho}\right), \rho \rightarrow 0, \quad f_{k k}^{(v)}(x, \rho)=O(1), \rho \rightarrow \mu, \quad \rho \in \overline{\Omega_{+}} .
$$

Proof. According to (16), (30) and Lemma 6 it is sufficient to prove that

$$
\begin{array}{ll}
\frac{1}{a(\rho)} \sum_{j \neq k} e_{j}^{\prime}(0, \rho) \prod_{l \neq k, j} e_{l}(0, \rho)=O\left(\frac{1}{\rho}\right), & \rho \rightarrow 0, \quad \rho \in \overline{\Omega_{+}}, \\
\frac{1}{a(\rho)} \sum_{j \neq k} e_{j}^{\prime}(0, \rho) \prod_{l \neq k, j} e_{l}(0, \rho)=O(1), \quad \rho \rightarrow \mu, \quad \rho \in \overline{\Omega_{+} .}
\end{array}
$$

We note that one cannot apply Lemma 6 directly, because in (57) $j$ can be chosen only from $\{p+1, \ldots, m\}$, while in (65), (66) we have $j=\overline{1, m} \backslash k$. Using (20), (23) we get

$$
\frac{1}{a(\rho)} \sum_{j \neq k} e_{j}^{\prime}(0, \rho) \prod_{l \neq k, j} e_{l}(0, \rho)=\frac{2 i \rho}{e_{k}(0, \rho)}-\frac{e_{k}^{\prime}(0, \rho)}{e_{k}(0, \rho) a(\rho)} \prod_{l \neq k} e_{l}(0, \rho) .
$$

Since $e_{k}(x, \rho) \neq 0$ for $\rho \in \mathbb{R}^{*}$, (66) follows solely from Lemma 6. As for (65) one should also recall that (see [27])

$$
\frac{\rho}{e_{k}(0, \rho)}=O(1), \quad \rho \rightarrow 0, \quad \rho \in \overline{\Omega_{+}} .
$$

Denote

$$
\varphi_{k}\left(\rho_{n}\right):=\operatorname{Res}_{\rho=\rho_{n}} f_{k}(\rho), \quad k=\overline{1, m}, \quad n=\overline{1, N} .
$$

Theorem 4 yields that nonzero functions $\varphi_{k}\left(\rho_{n}\right)=\left[\varphi_{k j}\left(x, \rho_{n}\right)\right]_{j=\overline{1, m}}$ are eigenfunctions related to the eigenvalue $\lambda_{n}=\rho_{n}^{2} \in \Lambda_{1}$ and hence we have

$$
\varphi_{k k}\left(x, \rho_{n}\right)=\alpha_{k n} e_{k}\left(x, \rho_{n}\right), \quad k=\overline{1, m}, \quad n=\overline{1, N} .
$$


The numbers $\alpha_{k n}$ are called norming constants associated with the edge $\varepsilon_{k}$. We note that if $\dot{a}\left(\rho_{n}\right) \neq 0$, then

$$
\alpha_{k n}=\frac{d_{k n}}{\dot{a}\left(\rho_{n}\right)} .
$$

Definition 2. The eigenvalue $\lambda_{n}$ is called invisible from the edge $\varepsilon_{j}, j \in\{1, \ldots, m\}$, if $y_{j}(x) \equiv 0$, for each eigenfunction $y=\left[y_{i}(x)\right]_{i=\overline{1, m}}$ related to $\lambda_{n}$; otherwise it is called visible from $\varepsilon_{j}$.

Denote by $\Lambda_{1}^{\prime}$ the set of all eigenvalues that are visible from at least one infinite edge $\varepsilon_{j}$, $j \in\{p+1, \ldots, m-1\}$. By virtue of Theorem $2 \Lambda_{1}^{\prime} \subset \Lambda_{1}$, and hence $\Lambda_{1}^{\prime}$ has the form

$$
\Lambda_{1}^{\prime}=\left\{\lambda_{n_{s}}\right\}_{s=\overline{1, N^{\prime}}}
$$

For $\lambda_{n} \notin \Lambda_{1}^{\prime}$ we obviously have $\alpha_{k n}=0, k=\overline{p+1, m-1}$.

Definition 3. The set

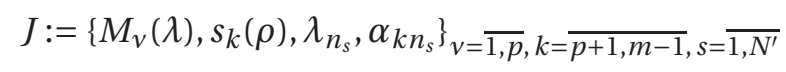

is called spectral-scattering data of $L$.

Remark 1. Denote by $\Lambda_{1}^{\prime \prime}$ the subset of $\Lambda_{1}^{\prime}$ consisting of all eigenvalues that are visible from at least one compact edge. Using (16), (19), (20) and Theorem 4 one can show that if $\lambda_{n}$ is visible from some compact edge $\varepsilon_{j}, j \in\{1, \ldots, p\}$, then $\lambda_{n}$ is a pole of the Weyl function $M_{j}(\lambda)$. Thus, the set $\Lambda_{1}^{\prime \prime}$ is completely determined by specification of $\left\{M_{v}(\lambda)\right\}_{v=1, p}$. Therefore in $J$ it is sufficient to specify among $\lambda_{n_{s}}, s=\overline{1, N^{\prime}}$, only those eigenvalues that belong to $\Lambda_{1}^{\prime} \backslash \Lambda_{1}^{\prime \prime}$, i.e. invisible from compact edges.

\section{Inverse problem. Uniqueness theorem}

The inverse problem is formulated as follows.

Inverse Problem 1. Given $J$, find $q$ and $H$.

We agree that together with $L$ we consider here and in the sequel a boundary value problem $\tilde{L}=L(\tilde{q}, \tilde{H})$ of the same form but with other coefficients. If a certain symbol $\gamma$ denotes an object corresponding to $L$, then this symbol with tilde $\tilde{\gamma}$ denotes the analogous object related to $\tilde{L}$.

Theorem 5. If $J=\tilde{J}$, then $L=\tilde{L}$, i.e. $q_{j}(x) \stackrel{\text { a.e. }}{=} \tilde{q}_{j}(x), j=\overline{1, m}, H_{j}=\tilde{H}_{j}, j=\overline{1, p}$. Thus, the specification of the spectral-scattering data J uniquely determines the coefficients of $L$. 
Proof. (i) Fix $k \in\{1, \ldots, p\}$. Let us show that $M_{k}(\lambda) \equiv \tilde{M}_{k}(\lambda)$ implies $H_{k}=\tilde{H}_{k}$ and $q_{k}(x)=\tilde{q}_{k}(x)$ a.e. on $(0,1)$. Define the matrix $P(x, \lambda)=\left[P_{v j}(x, \lambda)\right]_{v, j=1,2}$ by the formula

$$
P(x, \lambda)\left[\begin{array}{cc}
\tilde{\psi}_{k}(x, \lambda) & \tilde{\Psi}_{k k}(x, \lambda) \\
\tilde{\psi}_{k}^{\prime}(x, \lambda) & \tilde{\Psi}_{k k}^{\prime}(x, \lambda)
\end{array}\right]=\left[\begin{array}{cc}
\psi_{k}(x, \lambda) & \Psi_{k k}(x, \lambda) \\
\psi_{k}^{\prime}(x, \lambda) & \Psi_{k k}^{\prime}(x, \lambda)
\end{array}\right] .
$$

Using $\left\langle\psi_{k}(x, \lambda), \Psi_{k k}(x, \lambda)\right\rangle \equiv-1$, we calculate

$$
\left.\begin{array}{l}
P_{v 1}(x, \lambda)=\Psi_{k k}^{(v-1)}(x, \lambda) \tilde{\psi}_{k}^{\prime}(x, \lambda)-\psi_{k}^{(v-1)}(x, \lambda) \tilde{\Psi}_{k k}^{\prime}(x, \lambda), \\
P_{v 2}(x, \lambda)=\psi_{k}^{(v-1)}(x, \lambda) \tilde{\Psi}_{k k}(x, \lambda)-\Psi_{k k}^{(v-1)}(x, \lambda) \tilde{\psi}_{k}(x, \lambda) .
\end{array}\right\}
$$

Moreover, formula (69) in particular gives

$$
\psi_{k}(x, \lambda)=P_{11}(x, \lambda) \tilde{\psi}_{k}(x, \lambda)+P_{12}(x, \lambda) \tilde{\psi}_{k}^{\prime}(x, \lambda) .
$$

By virtue of (6), (10), (16)-(19), (23), (25), (70) and the asymptotis of the functions $C_{k}^{(v)}(x, \lambda)$, $S_{k}^{(v)}(x, \lambda), v=0,1$, (see [31]) we have

$$
P_{11}(x, \lambda)=1+O\left(\frac{1}{\rho}\right), \quad P_{12}(x, \lambda)=O\left(\frac{1}{\rho^{2}}\right), \quad \rho \in \Omega_{\varepsilon},|\rho| \rightarrow \infty,
$$

for each fixed $\varepsilon>0$. On the other hand, according to (21) and (70),

$$
\begin{aligned}
& P_{11}(x, \lambda)=\theta_{k}(x, \lambda) \tilde{\psi}_{k}^{\prime}(x, \lambda)-\tilde{\theta}_{k}^{\prime}(x, \lambda) \psi_{k}(x, \lambda)+\left(M_{k}(\lambda)-\tilde{M}_{k}(\lambda)\right) \psi_{k}(x, \lambda) \tilde{\psi}_{k}^{\prime}(x, \lambda), \\
& P_{12}(x, \lambda)=\psi_{k}(x, \lambda) \tilde{\theta}_{k}(x, \lambda)-\tilde{\psi}_{k}(x, \lambda) \theta_{k}(x, \lambda)+\left(\tilde{M}_{k}(\lambda)-M_{k}(\lambda)\right) \psi_{k}(x, \lambda) \tilde{\psi}_{k}(x, \lambda) .
\end{aligned}
$$

Thus, if $M_{k}(\lambda) \equiv \tilde{M}_{k}(\lambda)$, then for each fixed $x$ the functions $P_{11}(x, \lambda), P_{12}(x, \lambda)$ are entire in $\lambda$ of order $r \leq 1 / 2$. By virtue of the Phragmen-Lindelöf and Liouville theorems formulae (72) yield $P_{11}(x, \lambda) \equiv 1, P_{12}(x, \lambda) \equiv 0$. Substituting this into (71) we get $\psi_{k}(x, \lambda) \equiv \tilde{\psi}_{k}(x, \lambda)$ for all $x \in[0,1]$ and consequently $H_{k}=\tilde{H}_{k}$ and $q_{k}(x)=\tilde{q}_{k}(x)$ a.e. on $(0,1)$.

(ii) Fix $k \in\{p+1, \ldots, m-1\}$. Denote

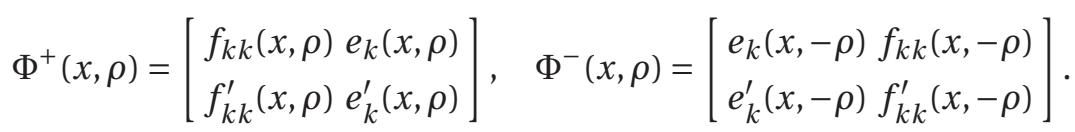

Lemma 7. For $\rho \in \mathbb{R}_{1}^{*}$ the following relation holds:

$$
\Phi^{-}(x, \rho)=\Phi^{+}(x, \rho) V(\rho), \quad V(\rho)=\left[\begin{array}{cc}
1 & s_{k}(-\rho) \\
-s_{k}(\rho) & 1-s_{k}(-\rho) s_{k}(\rho)
\end{array}\right] .
$$

Proof. According to (31) the functions $f_{k k}(x, \rho), e_{k}(x, \rho)$ form a fundamental system of solutions on the edge $\varepsilon_{k}$ if $f_{k}(x, \rho)$ exists - in particular, for $\rho \in \mathbb{R}_{1}^{*}$. Thus, we have

$$
\left.\begin{array}{l}
e_{k}(x,-\rho)=v_{11}(\rho) f_{k k}(x, \rho)+v_{21}(\rho) e_{k}(x, \rho) \\
f_{k k}(x,-\rho)=v_{12}(\rho) f_{k k}(x, \rho)+v_{22}(\rho) e_{k}(x, \rho) .
\end{array}\right\}
$$


Let us calculate the coefficients $v_{v j}(\rho)$. According to (11), (35), (75) we arrive at

$$
\begin{aligned}
& 2 i \rho v_{11}(\rho)=\left\langle e_{k}(x,-\rho), e_{k}(x, \rho)\right\rangle=2 i \rho, \\
& 2 i \rho v_{21}(\rho)=\left\langle f_{k k}(x, \rho), e_{k}(x,-\rho)\right\rangle=-2 i \rho s_{k}(\rho), \\
& 2 i \rho v_{12}(\rho)=\left\langle f_{k k}(x,-\rho), e_{k}(x, \rho)\right\rangle=2 i \rho s_{k}(-\rho), \\
& 2 i \rho v_{22}(\rho)=\left\langle f_{k k}(x, \rho), f_{k k}(x,-\rho)\right\rangle=2 i \rho\left(1-s_{k}(\rho) s_{k}(-\rho)\right),
\end{aligned}
$$

whence we get

$$
v_{11}(\rho)=1, \quad v_{12}(\rho)=s_{k}(-\rho), \quad v_{21}(\rho)=-s_{k}(\rho), \quad v_{22}(\rho)=1-s_{k}(-\rho) s_{k}(\rho),
$$

which together with (75) give (74).

Let us define the matrix $P^{+}(x, \rho)=\left[P_{v j}^{+}(x, \rho)\right]_{v, j=1,2}, \rho \in \overline{\Omega_{+}}$, by the formula

$$
P^{+}(x, \rho) \tilde{\Phi}^{+}(x, \rho)=\Phi^{+}(x, \rho) .
$$

By virtue of (31), (73), this yields

$$
\begin{gathered}
P_{v 1}^{+}(x, \rho)=\frac{1}{2 i \rho}\left(f_{k k}^{(v-1)}(x, \rho) \tilde{e}_{k}^{\prime}(x, \rho)-e_{k}^{(v-1)}(x, \rho) \tilde{f}_{k k}^{\prime}(x, \rho)\right), \\
P_{v 2}^{+}(x, \rho)=\frac{1}{2 i \rho}\left(e_{k}^{(v-1)}(x, \rho) \tilde{f}_{k k}(x, \rho)-f_{k k}^{(v-1)}(x, \rho) \tilde{e}_{k}(x, \rho)\right), \\
e_{k}(x, \rho)=P_{11}^{+}(x, \rho) \tilde{e}_{k}(x, \rho)+P_{12}^{+}(x, \rho) \tilde{e}_{k}^{\prime}(x, \rho) .
\end{gathered}
$$

Using (6), (10), (16), (17), (25), (30), (77) and the asymptotics of $C_{k}^{(v)}(x, \lambda), S_{k}^{(v)}(x, \lambda), v=0,1$, (see [31]) we get

$$
\left.\begin{array}{l}
P_{11}^{+}(x, \rho)=1+o\left(\frac{1}{\rho}\right), \\
P_{12}^{+}(x, \rho)=O\left(\frac{1}{\rho^{2}}\right),
\end{array}\right\} \quad|\rho| \rightarrow \infty, \rho \in \Omega_{\varepsilon} .
$$

Analogously we define the matrix $P^{-}(x, \rho)=\left[P_{v j}^{-}(x, \rho)\right]_{v, j=1,2}, \rho \in \overline{\Omega_{-}}$, by the formula

$$
P^{-}(x, \rho) \tilde{\Phi}^{-}(x, \rho)=\Phi^{-}(x, \rho) .
$$

Obviously, we have

$$
P^{-}(x, \rho)=P^{+}(x,-\rho) .
$$

Since $\tilde{s}_{k}(\rho)=s_{k}(\rho)$, formulae (74), (76), (80) give for $\rho \in \mathbb{R}_{1}^{*}$,

$$
\begin{aligned}
P^{-}(x, \rho) & =\Phi^{-}(x, \rho)\left(\tilde{\Phi}^{-}(x, \rho)\right)^{-1}=\Phi^{+}(x, \rho) V(\rho)\left(\tilde{\Phi}^{+}(x, \rho) \tilde{V}(\rho)\right)^{-1}= \\
& \left.=\Phi^{+}(x, \rho) V(\rho)(\tilde{V}(\rho))^{-1}\left(\tilde{\Phi}^{+}(x, \rho)\right)^{-1}=\Phi^{+}(x, \rho)\left(\tilde{\Phi}^{+} x, \rho\right)\right)^{-1}=P^{+}(x, \rho),
\end{aligned}
$$


and consequently for each fixed $x \geq 0$ the function $P^{-}(x, \rho)$ is an analytic continuation in $\Omega_{-}$ of $P^{+}(x, \rho)$. Thus, using (77), Corollary 3 and Definition 2 we conclude that the function

$$
P(x, \rho):=\left\{\begin{array}{l}
P^{+}(x, \rho), \rho \in \overline{\Omega_{+}}, \\
P^{-}(x, \rho), \rho \in \Omega_{-},
\end{array}\right.
$$

is meromorphic with at most simple poles in the points $\pm \rho_{n_{s}}, s=\overline{1, N^{\prime}}, \pm \tilde{\rho}_{n_{s}}, s=\overline{1, \tilde{N}^{\prime}}$, and with an at most second-order pole in the origin. Since $\tilde{N}^{\prime}=N^{\prime}, \tilde{\rho}_{n_{s}}=\rho_{n_{s}}, \tilde{\alpha}_{k n_{s}}=\alpha_{k n_{s}}, s=$ $\overline{1, N^{\prime}}$, according to (67), (68) we have

$$
\begin{aligned}
\operatorname{Res}_{\rho=\rho_{n_{s}}} P_{v 1}^{+}(x, \rho) & =\frac{1}{2 i \rho_{n_{s}}}\left(\varphi_{k k}^{(v-1)}\left(x, \rho_{n_{s}}\right) \tilde{e}_{k}^{\prime}\left(x, \rho_{n_{s}}\right)-e_{k}^{(v-1)}\left(x, \rho_{n_{s}}\right) \tilde{\varphi}_{k k}^{\prime}\left(x, \rho_{n_{s}}\right)\right) \\
& =\frac{\alpha_{k n_{s}}-\tilde{\alpha}_{k n_{s}}}{2 i \rho_{n_{s}}} e_{k}^{(v-1)}\left(x, \rho_{n_{s}}\right) \tilde{e}_{k}^{\prime}\left(x, \rho_{n_{s}}\right)=0, \\
\operatorname{Res}_{\rho=\rho_{n_{s}}} P_{v 2}^{+}(x, \rho) & =\frac{1}{2 i \rho_{n_{s}}}\left(\tilde{\varphi}_{k k}\left(x, \rho_{n_{s}}\right) e_{k}^{(v-1)}\left(x, \rho_{n_{s}}\right)-\tilde{e}_{k}\left(x, \rho_{n_{s}}\right) \varphi_{k k}^{(v-1)}\left(x, \rho_{n_{s}}\right)\right) \\
& =\frac{\tilde{\alpha}_{k n_{s}}-\alpha_{k n_{s}}}{2 i \rho_{n_{s}}} \tilde{e}_{k}\left(x, \rho_{n_{s}}\right) e_{k}^{(v-1)}\left(x, \rho_{n_{s}}\right)=0 .
\end{aligned}
$$

Thus, according to (81) the function $\rho^{2} P(x, \rho)$ is entire in $\rho$ of order $r \leq 1$. By virtue of (79), (81), the Phragmen-Lindelöf and Liouville theorems we get

$$
P_{11}^{+}(x, \rho) \equiv 1+\frac{C_{1}}{\rho^{2}}, \quad P_{12}^{+}(x, \rho) \equiv \frac{C_{2}}{\rho^{2}}, \quad C_{1}, C_{2}-\text { const },
$$

which together with (78) give

$$
e_{k}(x, \rho)=\tilde{e}_{k}(x, \rho)+\frac{1}{\rho^{2}}\left(C_{1} \tilde{e}_{k}(x, \rho)+C_{2} \tilde{e}_{k}^{\prime}(x, \rho)\right) .
$$

By virtue of continuity of $e_{k}(x, \cdot)$ in $\overline{\Omega_{+}}$we have

$$
C_{1} \tilde{e}_{k}(x, 0)+C_{2} \tilde{e}_{k}^{\prime}(x, 0) \equiv 0,
$$

which gives $C_{1}=0$. Indeed, according to (8) we have $\tilde{e}_{k}(x, 0) \not \equiv 0$ and hence $C_{1} \neq 0$ yields $C_{2} \neq 0$. Then (83) gives $\tilde{q}_{k}(x)=\left(C_{1} / C_{2}\right)^{2} \neq 0$, which contradicts $\tilde{q}_{k}(x) \in L(0, \infty)$.

Suppose that $C_{2} \neq 0$. Then by virtue of (83) we have $\tilde{e}_{k}^{\prime}(x, 0) \equiv 0$ and consequently $\tilde{q}_{k}(x)=$ 0 . Thus, $\tilde{e}_{k}(x, \rho)=\exp (i \rho x)$, which together with (82) give

$$
e_{k}(x, \rho)=\tilde{e}_{k}(x, \rho)+\frac{i C_{2}}{\rho} \exp (i \rho x) .
$$

Using again the continuity of $e(x, \cdot)$ we infer that $C_{2}=0$ and arrive at a contradiction. Thus, we get $C_{1}=C_{2}=0$ and according to (82) $e_{k}(x, \rho) \equiv \tilde{e}_{k}(x, \rho)$, which, in turn, gives $q_{k}(x)=\tilde{q}_{k}(x)$ a.e. on $(0, \infty), k=\overline{p+1, m-1}$. 
It remains to prove that $q_{m}(x)=\tilde{q}_{m}(x)$. The function $M(\lambda):=e_{m}(0, \rho)\left(e_{m}^{\prime}(0, \rho)\right)^{-1}$ is the Weyl function of the boundary value problem for equation (1) (for $j=m$ ) with the boundary condition $y_{m}^{\prime}(0)=0$. Thus, it is sufficient to prove that $\hat{M}(\lambda) \equiv 0$ (see [31]). By virtue of (15), (19) we have

$$
M(\lambda)=\frac{\Psi_{1 m}(0, \lambda)}{\Psi_{1 m}^{\prime}(0, \lambda)}
$$

According to the matching conditions (2) and representation (21) we have

$$
\begin{aligned}
& \Psi_{1 m}(0, \lambda)=\Psi_{11}(0, \lambda)=\theta_{1}(0, \lambda)+M_{1}(\lambda) \psi_{1}(0, \lambda), \\
& \Psi_{1 m}^{\prime}(0, \lambda)=-\theta_{1}^{\prime}(0, \lambda)-M_{1}(\lambda) \psi_{1}^{\prime}(0, \lambda)-\sum_{j=2}^{m-1} \Psi_{1 j}^{\prime}(0, \lambda) .
\end{aligned}
$$

By virtue of (15), (19) we get

$$
\Psi_{11}(0, \lambda)=\frac{1}{\Delta(\lambda)} \prod_{l=2}^{m} e_{l}(0, \rho), \quad \Psi_{1 j}^{\prime}(0, \lambda)=\frac{e_{j}^{\prime}(0, \rho)}{\Delta(\lambda)} \prod_{l \neq 1, j} e_{l}(0, \rho), \quad j \neq 1,
$$

whence we find

$$
\frac{e_{m}(0, \rho)}{\Delta(\lambda)}=\Psi_{11}(0, \lambda)\left(\prod_{l=2}^{m-1} e_{l}(0, \rho)\right)^{-1}, \quad \Psi_{1 j}^{\prime}(0, \lambda)=\Psi_{11}(0, \lambda) \frac{e_{j}^{\prime}(0, \rho)}{e_{j}(0, \rho)},
$$

which together with (86) gives

$$
\Psi_{1 m}^{\prime}(0, \lambda)=-\theta_{1}^{\prime}(0, \lambda)-M_{1}(\lambda) \psi_{1}^{\prime}(0, \lambda)-\left(\theta_{1}(0, \lambda)+M_{1}(\lambda) \psi_{1}(0, \lambda)\right) \sum_{j=2}^{m-1} \frac{e_{j}^{\prime}(0, \rho)}{e_{j}(0, \rho)}
$$

Using (84), (85), (87) we arrive at $\hat{M}(\lambda) \equiv 0$.

\section{References}

[1] E. Montrol, Quantum theory on a network, J. Math. Phys., 11(1970), 635-648.

[2] M. D. Faddeev and B. S. Pavlov, Model of free electrons and the scattering problem, Teor. Math. Fiz., 55 (1983), 257-269 (Russian); English transl. in Theor. Math. Phys., 55(1983), 485-492.

[3] J.-P. Roth, Spectre du laplacien sur un graphe, C. R. Acad. Sci. Paris, 296(1983), 783-795.

[4] S. Nicaise, Some results on spectral theory over networks, applied to nerve impulse transmission, Lect. Notes Math. 1771, Springer, 1985, 532-541.

[5] J. von Below, Sturm-Liouville eigenvalue problem on networks, Math. Methods Appl. Sci., 10(1988), $383-395$.

[6] J. E. Langese, Leugering G. and Schmidt J.P.G., Modelling, analysis and control of dynamic elastic multi-link structures, Birkhäuser, Boston, 1994.

[7] F. Ali-Mehmeti, Nonlinear waves in networks, Math. Research, 80(1994).

[8] P. Exner, Contact interactions on graph superlattices, J. Phys. A: Math. Gen., 29(1996), 87-102.

[9] T. Kottos and U. Smilansky, Quantum chaos on graphs, Phys. Rev. Lett., 79(1997), 4794-4797.

[10] J. Tautz, M. Lindauer and D.C. Sandeman, Transmission of vibration across honeycombs and its detection by bee leg receptors, J. Exp. Biol., 199(1999), 2585-2594. 
[11] B. Dekoninck and S. Nicaise, The eigenvalue problem for networks of beams, Linear Algebra Appl., 314(2000), 165-189.

[12] J. Desbois, Spectral determinant of Schrödinger operators on graphs, J. Phys. A: Math. Gen., 33(2000), L63-7.

[13] G. Berkolaiko, E. Bogomolny and J. Keating, Star graphs and Seba billiards, J. Phys. A: Math. Gen., 34 (2001), 335-350.

[14] A. Sobolev and M. Solomyak, Schrödinger operator on homogeneous metric trees: spectrum in gaps, Rev. Math. Phys., 14(2002), 421-467.

[15] Yu. V. Pokornyi and A. V. Borovskikh, Differential equations on networks (geometric graphs), J. Math. Sci. (N.Y.), 119(2004), 691-718.

[16] Yu. Pokornyi and V. Pryadiev, The qualitative Sturm-Liouville theory on spatial networks, J. Math. Sci. (N.Y.), 119(2004), 788-835.

[17] M. I. Belishev, Boundary spectral inverse problem on a class of graphs (trees) by the BC method, Inverse Problems, 20(2004), 647-672.

[18] V. A. Yurko, Inverse spectral problems for Sturm-Liouville operators on graphs, Inverse Problems, 21(2005), 1075-1086.

[19] B. M. Brown and R. Weikard, A Borg-Levinson theorem for trees, Proc. R. Soc. Lond. Ser. A Math. Phys. Eng. Sci., 461(2005), no.2062, 3231-3243.

[20] G. Freiling and V. A. Yurko, Inverse problems for differential operators on graphs with general matching conditions, Appl. Anal., 86(2007), 653-667.

[21] V. A. Yurko, Inverse problems for Sturm-Liouville operators on bush-type graphs, Inverse Problems, 25(2009), no.10, 105008, 14pp.

[22] V. A. Yurko, Uniqueness of recovering Sturm-Liouville operators on A-graphs from spectra, Results in Mathematics, 55(2009), 199-207.

[23] V. A. Yurko, Inverse spectral problems for differential operators on arbitrary compact graphs, J. Inv. Ill-Posed Problems, 18(2010), 245-261.

[24] G. Freiling and V. A. Yurko, Inverse spectral problems for Sturm-Liouville operators on noncompact trees, Results in Mathematics, 50(2007), 195-212.

[25] V. A. Yurko, Inverse problems for differential operators of any order on trees, Matemat. Zametki, 83(2008), 139-152; English transl. in Math. Notes, 83(2008), 125-137.

[26] V. A. Yurko, Inverse spectral problems for arbitrary order differential operators on noncompact trees, J. Inverse Ill-Posed Probl. 20 (2012), no.1, 111-131.

[27] V. A. Marchenko, Sturm-Liouville operators and their applications, Naukova Dumka, Kiev, 1977; English transl. in Birkhäuser, 1986.

[28] B. M. Levitan, Inverse Sturm-Liouville problems. Nauka, Moscow, 1984; English transl., VNU Sci. Press, Utrecht, 1987.

[29] L. D. Faddeev, On a connection the S-matrix with the potential for the one-dimensional Schrödinger operator, Dokl. Akad. Nauk SSSR, 121(1958), 63-66.

[30] P. Deift and E. Trubowitz, Inverse scattering on the line, Comm. Pure Appl. Math., 32(1979), 121-251.

[31] G. Freiling and V. A. Yurko, Inverse Sturm-Liouville Problems and their Applications. NOVA Science Publishers, New York, 2001.

[32] N. I. Gerasimenko, Inverse scattering problem on a noncompact graph, Teoret. Mat. Fiz., 74(1988), 187-200 (Russian); English transl. in Theor. Math. Phys., 75 (1988) 460-470.

[33] M. Harmer, Inverse scattering on matrices with boundary conditions, J. Phys. A, 38(2005), 4875-4885.

[34] I. Trooshin, V. Marchenko and K. Mochizuki, Inverse scattering on a graph containing circle, Analytic Methods of Analysis and DEs: AMADE 2006 (Cambridge: Cambridge Science), 237-243.

[35] G. Freiling and M. Ignatyev, Spectral analysis for Sturm-Liouville operator on sun-type graphs, Inverse Problems, 27(2011), 095033 (17pp).

[36] M. Ignatyev, Inverse scattering problem for Sturm-Liouville operator on one-vertex noncompact graph with a cycle, Tamkang J. Math., 42 (2011), 365-384. 
[37] P. Kurasov and F. Stenberg, On the inverse scattering problem on branching graphs, J. Phys. A: Math. Gen., 35(2002), 101-121.

[38] J. Boman and P. Kurasov, Symmetries of quantum graphs and the inverse scattering problem, Adv. Appl. Math., 35(2005), 58-70.

[39] V. A. Yurko, Method of Spectral Mappings in the Inverse Problem Theory, Inverse and Ill-posed Problems Series, VSP, Utrecht, 2002.

[40] S. A. Buterin and G. Freiling, Inverse scattering problem for the Sturm-Liouville operator on a noncompact star-type graph, Schriftenreihe des Fachbereichs Mathematik, SM-DU-725, Universitaet Duisburg-Essen, 2011, 17pp.

[41] S. A. Buterin, Inverse scattering problem for the Sturm-Liouville operator on a noncompact star-type graph with general matching conditions, Spectral and Evolutional Problems, 21(2011), 77-87 (Russian).

[42] M. A. Naimark, Linear Differential Operators. 2nd ed., Nauka, Moscow, 1969; English transl. of 1st ed., Parts I,II, Ungar, New York, 1967, 1968.

Department of Mathematics, Saratov State University, Astrakhanskaya 83, Saratov 410012, Russia.

E-mail: buterinsa@info.sgu.ru

Department of Mathematics, Duisburg-Essen University, Forsthausweg 2, Duisburg 47057, Germany.

E-mail: gerhard.freiling@uni-due.de 